\title{
NLO Cross Sections for the LHC using GOLEM: Status and Prospects
}

\section{Thomas Binoth}

The University of Edinburgh, School of Physics, Edinburgh EH93JZ, UK

\section{Gavin Cullen}

The University of Edinburgh, School of Physics, Edinburgh EH9 3JZ, UK

E-mail: g.j.cullen@sms.ed.ac.uk

\section{Nicolas Greiner}

Institute for Theoretical Physics, University of Zürich, CH-8057 Zürich, Switzerland

E-mail: greiner@physik.uzh.ch

\section{Alberto Guffanti}

Physikalisches Institut, Albert-Ludwigs-Universität Freiburg, 79104 Freiburg i. Br, Germany

E-mail: alberto.guffanti@physik.uni-freiburg.de

\section{Jean-Philippe Guillet}

LAPTH, Annecy-le-Vieux 74951, France

E-mail: guilletelapp.in2p3.fr

\section{Gudrun Heinrich}

IPPP, Department of Physics, University of Durham, Durham DH1 3LE, UK

E-mail: gudrun.heinrichedurham.ac.uk

\section{Stefan Karg}

Institut für Theoretische Physik E, RWTH Aachen, 52056 Aachen, Germany

E-mail: karg@physik.rwth-aachen.de

\section{Nikolas Kauer}

Department of Physics, Royal Holloway, University of London, Egham TW20 0EX, UK School of Physics and Astronomy, University of Southampton, Southampton SO17 1BJ, UK

E-mail: n.kauer@rhul.ac.uk

\section{Thomas Reiter*}

Nikhef, 1098 XG Amsterdam, The Netherlands

E-mail: thomasrenikhef.nI

\section{Jürgen Reuter}

Physikalisches Institut, Albert-Ludwigs-Universität Freiburg, 79104 Freiburg i. Br, Germany

E-mail: juergen.reuteraphysik.uni-freiburg.de 
In this talk we review the GOLEM approach to one-loop calculations and present an automated implementation of this technique. This method is based on Feynman diagrams and an advanced reduction of one-loop tensor integrals which avoids numerical instabilities. We have extended our one-loop integral library golem 95 with an automated one-loop matrix element generator to compute the virtual corrections of the process $q \bar{q} \rightarrow b \bar{b} b \bar{b}$. The implementation of the virtual matrix element has been interfaced with tree-level Monte Carlo programs to provide the full result for the above process.

RADCOR 2009 - 9th International Symposium on Radiative Corrections (Applications of Quantum Field Theory to Phenomenology)

October 25-30 2009

Ascona, Switzerland

${ }^{*}$ Speaker. 


\section{Overview}

The successful start of the LHC and its first data taking mark the dawn of a new era in particle physics. Very soon we will be able to explore the energy scale of electro-weak symmetry breaking which enables us to either confirm the Standard Model as a low energy theory of particle physics or to discover new particles guiding us to extensions of the Standard Model. No matter what the outcome of the experiment will be, a successful interpretation of the data will require a large number of predictions calculated at least to Next-To-Leading Order (NLO) in QCD. Some of these reactions involve up to four particles in the final state $[1,2,3,4]$.

The computation of NLO corrections to processes involving many final state particles are cumbersome and time consuming. The time required for a single calculation - without the use of automated programs - often coincides with the duration of a Ph.D. Considering the fast experimental progress it is very likely that already within the next two or three years the physics results of the LHC will raise the demand for precision calculations within and beyond the Standard Model for many multileg processes. Only the automatisation of NLO calculations will allow us to keep pace with the requirements set by the experiments.

We argue that the automatisation of the computation of NLO cross-sections will also improve the possibility of comparing results from different implementations, especially if the tools are made public and common conventions are used for input/output and interfacing to external programs are in use. A first step in this direction has been made through the Binoth Les Houches Accord [5]. This accord exploits the modular structure of NLO calculations and proposes the reflection of this structure in the implementation of such calculations as computer programs.

Any QCD cross-section at NLO can be written in the form

$$
\sigma_{2 \rightarrow N}^{\mathrm{NLO}}=\sigma_{2 \rightarrow N}^{\mathscr{B}}+\sigma_{2 \rightarrow N}^{\mathscr{V}}+\sigma_{2 \rightarrow N+1}^{\mathscr{R}}
$$

The first term on the right-hand side describes the Born-level cross-section calculated from the squared tree-level amplitude of the $2 \rightarrow N$ process. The virtual corrections $\sigma_{2 \rightarrow N}^{\mathscr{V}}$ stem from the interference term between tree-level and one-loop diagrams. The third term describes the real radiation of an extra, unobserved parton at tree-level.

The last two terms lead to singularities which can be regularised by introducing a non-integer dimension $n=4-2 \varepsilon$, yielding poles in $1 / \varepsilon$ which cancel only after both terms have been added up. For practical applications it is therefore convenient to introduce subtraction terms, such that both $\sigma_{2 \rightarrow N}^{\mathscr{V}}$ and $\sigma_{2 \rightarrow N+1}^{\mathscr{R}}$ are finite and can be integrated over phase space independently.

A full implementation of an NLO calculation can therefore be modularised into a MonteCarlo integrator for phase space integration, a tree-level matrix element generator for $\sigma_{2 \rightarrow N}^{\mathscr{B}}$ and $\sigma_{2 \rightarrow N+1}^{\mathscr{R}}$, a one-loop matrix element generator for $\sigma_{2 \rightarrow N}^{\mathscr{V}}$ and infrared subtraction terms. Typically the first two of these components are implemented in the same program. An overview of existing techniques and recent contributions to all of the modules can be found, for example, in [2] and [5].

Methods for computing $\sigma_{2 \rightarrow N}^{\mathscr{V}}$ can be classified in two groups. On the one hand there are unitarity based methods determining the coefficients of scalar one-loop integrals by exploiting analyticity of the amplitude; on the other hand there are Feynman diagrammatic techniques starting from tensor integrals, which are then reduced to simpler integrals which can be evaluated in a 
numerically stable way. Both techniques have led to automated implementations in recent years ([6, 7, 8, 9, 10,11] and $[12,13,14,15,16]$ respectively).

In Sections 2 and 3 we present the automated implementation of a method using Feynman diagrams for the calculation of $\sigma_{2 \rightarrow N}^{\mathscr{V}}$, the GOLEM method, based on the reduction scheme proposed in [17]. This implementation has been used to compute the NLO virtual corrections to $q \bar{q} \rightarrow b \bar{b} b \bar{b}$, which is one of the two partonic initial states contributing to the $p p \rightarrow b \bar{b} b \bar{b}$ process. This process constitutes an important background for Higgs searches in models beyond the Standard Model where Higgs bosons decay predominantly into $b$-quarks as discussed in $[18,19]$.

\section{The Generic Integral Form Factor Library golem95}

The Feynman diagrammatic approach to computation of one-loop corrections to processes with $\mathrm{N}$ external particles requires the evaluation of tensor integrals of rank $r$ which have the general form

$$
I_{\mathrm{N}}^{n ; \mu_{1} \ldots \mu_{r}}\left(a_{1}, \ldots, a_{r} ; S\right)=\int \frac{\mathrm{d}^{n} k}{i \pi^{n / 2}} \frac{q_{a_{1}}^{\mu_{1}} \cdots q_{a_{r}}^{\mu_{r}}}{\left(q_{1}^{2}-m_{1}^{2}+i \delta\right) \cdots\left(q_{\mathrm{N}}^{2}-m_{\mathrm{N}}^{2}+i \delta\right)},
$$

where $q_{i}=k+r_{i}$ and $S$ denotes the matrix $S_{i j}=\left(r_{i}-r_{j}\right)^{2}-m_{i}^{2}-m_{j}^{2}$.

It is well-known [20] that such a tensor integral can be expressed in terms of basis scalar integrals, but the reduction procedure introduces inverse Gram determinants $(\operatorname{det} G)$ in the coefficients of the expansions which can lead to numerical instabilities in certain regions of the phase space.

Therefore, in $[21,17]$ we have proposed a reduction scheme which allows to write any Npoint amplitude as a linear combination of basis integrals $\left(I_{2}, I_{3}^{n}, I_{3}^{n+2}, I_{4}^{n+2}, I_{4}^{n+4}\right)$ with and without Feynman parameters in the numerator, avoiding the introduction of inverse Gram determinants.

The evaluation of the basis functions can be performed by reducing them further to scalar integrals using recursion formulae. This further reduction, however, introduces Gram determinants in the coefficients which could lead to numerical instabilities in certain regions of the phase space.

Potentially dangerous regions are identified by the criterion $|\operatorname{det} G|<\Lambda|\operatorname{det} S|$ for a fixed cutoff $\Lambda \sim 10^{-5}$. In this regions the basis integrals are evaluated numerically without applying any further reduction.

For all integrals without internal masses we have worked out one-dimensional integral representations which can be evaluated by numerical integration.

This algorithm has been implemented in form of a Fortran 90 library, golem95, for massless internal propagators $\left(m_{i}=0\right)$ [16]. This version of the code has been made available for download ${ }^{1}$.

We have recently extended the library golem 95 to the case where internal masses are present. All infrared divergent integrals have been implemented explicitly. For evaluating the finite boxes and triangles the user needs to link the LoopTools library [12, 22, 23]. This "massive" version of the golem 95 library is currently in the testing phase and will be available shortly.

For integrals with internal masses, the option to evaluate the tensor integrals numerically prior to reduction in regions where the Gram determinant tends to zero, is not yet supported. However, one-dimensional integral representations valid for all possible kinematic configurations are under construction.

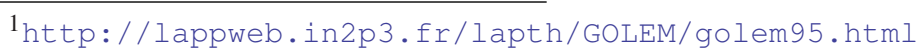




\section{Implementation of a One-Loop Matrix Element Generator}

Building upon golem95 as a loop-integral library, our next step was the construction of a matrix-element generator at the one-loop level. The computation is carried out projecting the amplitude onto helicity and colour structures. The virtual corrections can therefore be expressed as

$$
\mathrm{d} \sigma_{2 \rightarrow N}^{\mathscr{V}}=\frac{1}{n_{a} n_{b}} \sum_{\left\{\lambda_{i}\right\}, j, k} \mathscr{A}_{j}^{\mathscr{B}}\left(p_{a}^{\lambda_{a}}, p_{b}^{\lambda_{b}} ; p_{1}^{\lambda_{1}}, \ldots, p_{N}^{\lambda_{N}}\right)^{\dagger}\left\langle c_{j} \mid c_{k}\right\rangle \mathscr{A}_{k}^{\mathscr{V}}\left(p_{a}^{\lambda_{a}}, p_{b}^{\lambda_{b}} ; p_{1}^{\lambda_{1}}, \ldots, p_{N}^{\lambda_{N}}\right)+\text { h.c. }
$$

where $p_{i}^{\lambda_{i}}$ denotes the pair of momentum $p_{i}$ and helicity label $\lambda_{i}$ of the $i-$ th particle. The matrix $\left\langle c_{j} \mid c_{k}\right\rangle$ consists of the contractions of all colour basis tensors for a given process evaluating to rational functions in the number of colours $N_{C}$ and the normalisation constant $T_{R}$ of the generators. The constants $n_{a}$ and $n_{b}$ represent the averaging over spin and colour.

The one-loop amplitude $\sum_{k} \mathscr{A}_{k}\left|c_{k}\right\rangle$ consists of a sum of Feynman diagrams, which we generate using QGraf [24]. We use QGraf together with $\mathrm{LT}_{\mathrm{E}} \mathrm{X}$ and Axodraw [25] also for drawing the diagrams; the layout of the diagrams is determined using the algorithm of [26].

The expressions of the diagrams are then processed using Form [27] and the Form library spinney which we have developed for dealing with helicity spinors and $n$-dimensional Dirac and Lorentz algebra efficiently. Majorana spinors can also be dealt with thanks to the the implementation the flipping rules for spin lines as described in [28].

At the moment the GOLEM program can import CompHep [29] model files to perform Beyond the Standard Model computations. An interface to FeynRules [30] is under construction.

After the Form program has decomposed the diagram expression into colour structures and the tensor integrals are represented in terms of integral form factors as defined in golem95 [16], the resulting expressions are optimised and translated into Fortran 90 functions using the code generator haggies [31]. At this step the number of multiplications is minimised applying a Horner scheme and common subexpression elimination.

The generated Fortran 90 program is linked with golem 95 for the numerical evaluation of the tensor integral form factors. A future version of the program will support the Binoth Les Houches Accord [5] to facilitate the interfacing to Monte-Carlo generators.

\section{NLO Results for $q \bar{q} \rightarrow b \bar{b} b \bar{b}[32]$}

The setup described in the previous section has been used to compute the virtual corrections of the QCD corrections to $q \bar{q} \rightarrow b \bar{b} b \bar{b}$ in the limit $m_{b}=0$ and $m_{t} \rightarrow \infty$. We have compared the results with an independent implementation using FeynArts and FormCalc [12] to generate and simplify the diagrams, where the tensor integrals are algebraically reduced to scalar integrals using the procedure described in [17].

In order to compute $q \bar{q} \rightarrow b \bar{b} b \bar{b}$ at NLO accuracy the Born level cross-section, the real emission contribution and the infrared subtraction terms also need to be evaluated. Since we are only interested in a process with 4 tagged $b$-jets the relevant process for the real emission contribution is $q \bar{q} \rightarrow b \bar{b} b \bar{b} g$.

We have used MadGraph/MadEvent [33, 34] and MadDipole [35] to evaluate the treelike contributions and to perform the phase space integration. As an alternative setup, based on 
an extended version of the Whizard [36, 37] Monte Carlo generator with an implementation of infrared subtraction terms has been used to obtain an independent cross-check. For the infrared subtraction we have used Catani-Seymour dipoles [38] in both implementations including a phase space slicing parameter following [39].

To define a $b \bar{b} b \bar{b}$ event, we first we apply a $k_{T}$ jet algorithm [40] to decide if the gluon should be merged with a $b$-quark. If the gluon is merged we use the effective momentum $\tilde{p}_{b}=p_{b}+p_{g}$ as the momentum of the $b$-quark in the cuts and in the observables. Then we apply a $p_{T}$ cut of $p_{T}\left(b_{j}\right)>30 \mathrm{GeV}$ and a rapidity cut of $|\eta|<2.5$ to all $b$-quarks and a separation cut of $\Delta R>0.8$ to all pairs of $b$-quarks.

We sum over $q \in\{u, d, c, s\}$ and use the CTEQ6M parton distribution functions [41] with twoloop running of $\alpha_{s}$ for both Leading and Next-to-Leading Order computations. The centre of mass energy is set to $\sqrt{s}=14 \mathrm{GeV}$. In our results we use a fixed factorisation scale of $\mu_{F}=100 \mathrm{GeV}$; the renormalisation scale we set to $\mu_{0}=\sqrt{\sum_{j} p_{T}^{2}\left(b_{j}\right)}$.

In Figure 1 we show the invariant mass distribution of the system of the two $b$-pairs with highest $p_{T}$. The error bands have been obtained by varying the renormalisation scale $\mu_{R}=x \mu_{0}$ between $1 / 4<x<2$. The dashed line marks the leading order distribution for $x=1 / 2$, which turns out to be very similar to the NLO prediction for this value. The reduction of the uncertainty band due to scale variations clearly shows the importance of the NLO corrections for the precision of this calculation.

\section{Conclusion}

We have presented results obtained using the GOLEM method and recent progress in the implementation of an automated one-loop matrix element generator. A first important step towards this goal was the development of a one-loop integral library, golem95, which is currently being extended to the case of massive propagators. As a second step we have added a completely automated framework which generates efficient Fortran 90 code for the numerically stable evaluation of one-loop matrix elements from a set of Feynman rules. This framework includes the development of new tools such as an optimising code generator (haggies), and a Form library (spinney) for the treatment of helicity spinors and $n$-dimensional Dirac and Lorentz algebra. Finally, we have presented the complete NLO result for the process $q \bar{q} \rightarrow b \bar{b} b \bar{b}$, which is a subprocess of $p p \rightarrow b \bar{b} b \bar{b}$, an important background to Higgs searches beyond the Standard Model.

In the near future we plan to implement the interface to Monte-Carlo tools described in [5] and to make all parts of the program publicly available.

\section{References}

[1] N. E. Adam et al., 0803.1154 [hep-ph].

[2] Z. Bern et al. [NLO Multileg Working Group], 0803.0494 [hep-ph].

[3] C. Buttar et al., hep-ph/0604120.

[4] J. M. Campbell, J. W. Huston and W. J. Stirling, Rept. Prog. Phys. 70 (2007) 89 [hep-ph/0611148]. 


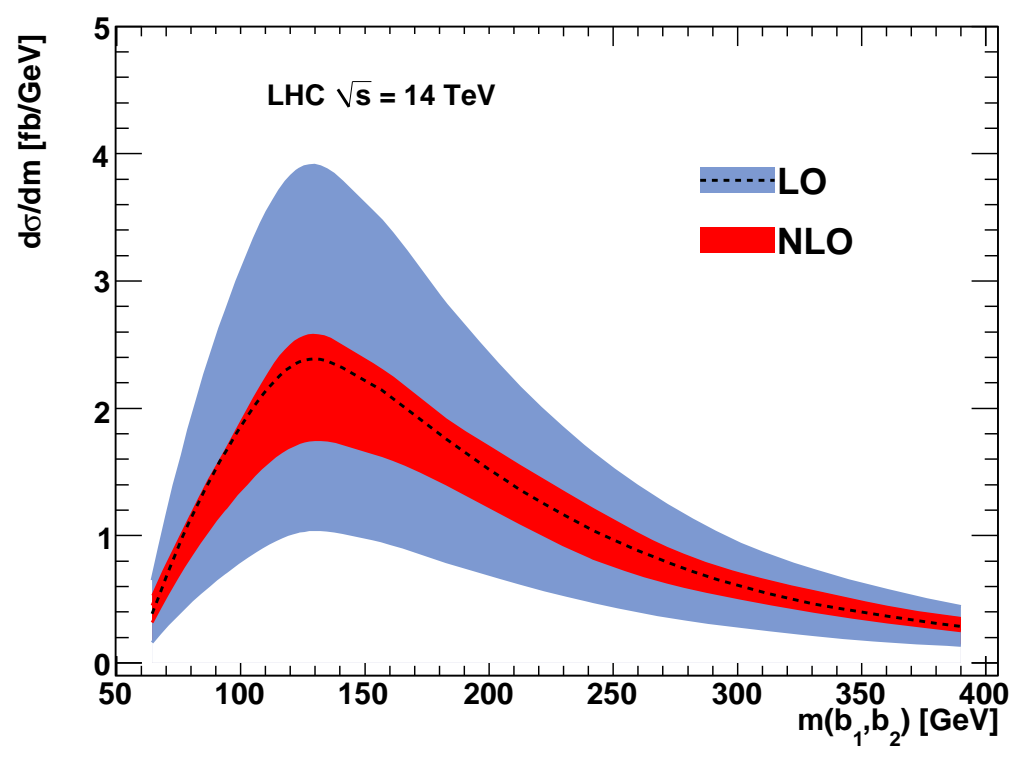

Figure 1: Invariant mass $\left(m_{b b}\right)$ distribution of the two leading $b$-quarks (see text). The error bands are obtained by varying the renormalisation scale $\mu_{R}=x \mu_{0}$ between $1 / 4<x<2$, where $\mu_{0}=\sqrt{\sum_{j} p_{T}^{2}\left(b_{j}\right)}$. The dashed line shows the value of the leading order prediction for $x=1 / 2$.

[5] T. Binoth et al., 1001.1307 [hep-ph].

[6] R. K. Ellis, W. T. Giele and Z. Kunszt, JHEP 0803 (2008) 0030708.2398 [hep-ph].

[7] G. Ossola, C. G. Papadopoulos and R. Pittau, JHEP 0803 (2008) 0420711.3596 [hep-ph].

[8] C. F. Berger et al., Phys. Rev. D 78 (2008) 0360030803.4180 [hep-ph].

[9] W. T. Giele and G. Zanderighi, JHEP 0806 (2008) 0380805.2152 [hep-ph].

[10] A. Lazopoulos, 0812.2998 [hep-ph]. 
[11] J. C. Winter and W. T. Giele, 0902.0094 [hep-ph].

[12] T. Hahn and M. Perez-Victoria, Comput. Phys. Commun. 118 (1999) 153 [hep-ph/9807565].

[13] Y. Kurihara, J. Fujimoto, T. Ishikawa, K. Kato, S. Kawabata, T. Munehisa and H. Tanaka, Nucl. Phys. B 654, 301 (2003) [hep-ph/0212216].

[14] G. Belanger, F. Boudjema, J. Fujimoto, T. Ishikawa, T. Kaneko, K. Kato and Y. Shimizu, Phys. Rept. 430 (2006) 117 [hep-ph/ 0308080$].$

[15] A. Bredenstein, A. Denner, S. Dittmaier and S. Pozzorini, 1001.4006 [hep-ph].

[16] T. Binoth, J. P. Guillet, G. Heinrich, E. Pilon and T. Reiter, Comput. Phys. Commun. 180 (2009) 2317 0810.0992 [hep-ph].

[17] T. Binoth, J. P. Guillet, G. Heinrich, E. Pilon and C. Schubert, JHEP 0510 (2005) 015 [hep-ph/0504267].

[18] R. Lafaye, D. J. . Miller, M. Muhlleitner and S. Moretti, [hep-ph/0002238].

[19] W. Krolikowski, 0803.2977 [hep-ph].

[20] D. B. Melrose, Nuovo Cim. 40 (1965) 181.

[21] T. Binoth, J. P. Guillet and G. Heinrich, Nucl. Phys. B 572 (2000) 361 [hep-ph/9911342].

[22] T. Hahn, Comput. Phys. Commun. 140, 418 (2001) [hep-ph/ 0012260 ].

[23] G. J. van Oldenborgh and J. A. M. Vermaseren, Z. Phys. C 46, 425 (1990).

[24] P. Nogueira, J. Comput. Phys. 105 (1993) 279.

[25] J. A. M. Vermaseren, Comput. Phys. Commun. 83 (1994) 45.

[26] T. Ohl, Comput. Phys. Commun. 90 (1995) 340 [hep-ph/ 9505351$].$

[27] J. A. M. Vermaseren, [math-ph/0010025].

[28] A. Denner, H. Eck, O. Hahn and J. Kublbeck, Nucl. Phys. B 387, 467 (1992).

[29] E. Boos et al. [CompHEP Collaboration], Nucl. Instrum. Meth. A 534, 250 (2004) [hep-ph/0403113].

[30] N. D. Christensen and C. Duhr, Comput. Phys. Commun. 180, 1614 (2009) 0806.4194 [hep-ph].

[31] T. Reiter, 0907.3714 [hep-ph].

[32] T. Binoth, N. Greiner, A. Guffanti, J. P. Guillet, T. Reiter and J. Reuter, 0910.4379 [hep-ph].

[33] F. Maltoni and T. Stelzer, JHEP 0302 (2003) 027 [hep-ph/ 0208156 ].

[34] J. Alwall et al., JHEP 0709 (2007) 0280706.2334 [hep-ph].

[35] R. Frederix, T. Gehrmann and N. Greiner, JHEP 0809 (2008) 1220808 . 2128 [hep-ph].

[36] W. Kilian, T. Ohl and J. Reuter, 0708.4233 [hep-ph].

[37] M. Moretti, T. Ohl and J. Reuter, arXiv:hep-ph/0102195.

[38] S. Catani and M. H. Seymour, Nucl. Phys. B 485 (1997) 291 [Erratum-ibid. B 510 (1998) 503] [hep-ph/9605323].

[39] Z. Nagy and Z. Trocsanyi, Phys. Lett. B 634, 498 (2006) [hep-ph/0511328].

[40] G. C. Blazey et al., [hep-ex/0005012].

[41] J. Pumplin, D. R. Stump, J. Huston, H. L. Lai, P. M. Nadolsky and W. K. Tung, JHEP 0207, 012 (2002) [hep-ph/0201195]. 